\title{
Impairments due to Burst-Mode Transmission in a Raman-based Long Reach PON Link
}

\author{
Kjær, Rasmus; Tafur Monroy, Idelfonso; Oxenløwe, Leif Katsuo; Jeppesen, Palle; Palsdottir, Bera
}

Published in:

I E E E Photonics Technology Letters

Link to article, DOI:

10.1109/LPT.2007.903768

Publication date:

2007

Document Version

Publisher's PDF, also known as Version of record

Link back to DTU Orbit

Citation (APA):

Kjær, R., Tafur Monroy, I., Oxenløwe, L. K., Jeppesen, P., \& Palsdottir, B. (2007). Impairments due to BurstMode Transmission in a Raman-based Long Reach PON Link. I E E E Photonics Technology Letters, 19(19), 1490-1492. https://doi.org/10.1109/LPT.2007.903768

\section{General rights}

Copyright and moral rights for the publications made accessible in the public portal are retained by the authors and/or other copyright owners and it is a condition of accessing publications that users recognise and abide by the legal requirements associated with these rights.

- Users may download and print one copy of any publication from the public portal for the purpose of private study or research.

- You may not further distribute the material or use it for any profit-making activity or commercial gain

- You may freely distribute the URL identifying the publication in the public portal 


\title{
Impairments Due to Burst-Mode Transmission in a Raman-Based Long-Reach PON Link
}

\author{
Rasmus Kjær, Idelfonso Tafur Monroy, Leif K. Oxenløwe, Palle Jeppesen, Member, IEEE, and Bera Palsdottir
}

\begin{abstract}
A recently proposed passive-optical-network (PON) link based on distributed Raman amplification is tested with disturbing burst-mode traffic. The resulting impairments are quantified through penalty measurements on a single surviving data channel as a function of the disturbing channel power. When the disturbing channels co- or counterpropagate with the data channel, penalties of less than $1 \mathrm{~dB}$ are found for disturbing input powers up to 7 and $11 \mathrm{dBm}$, respectively. The penalty is further reduced when a moderate amount of continuous-wave light is used to clamp the gain. The results indicate that the highly linear PON link is a promising candidate for long-reach feeder links in future, high-capacity PON systems with burst-mode traffic.
\end{abstract}

Index Terms-Burst-mode traffic, crosstalk, passive optical networks (PONs), Raman amplification.

\section{INTRODUCTION}

$\mathbf{T}$ HE long-reach, or super, passive optical network (PON) is a promising way to reduce the cost and increase the bandwidth of future access networks [1], [2]. The idea is to simplify the network architecture with a reduced number of network nodes, each connected to a number of high-capacity feeder links with up to $100-\mathrm{km}$ reach, thus extending the system reach far beyond the $20-\mathrm{km}$ reach of current PONs. The feeder link connects the metro core node to the local exchange in the access network (see Fig. 1). This link is an essential part of long-reach PON systems and its design can ultimately limit the maximum system reach, splitting ratio, and channel capacity.

To extend the reach of existing PONs, amplification must be employed to compensate for loss in the system. Due to packetbased traffic and changing bandwidth demands from end users, the employed amplifiers must be able to deal with fluctuating input powers and prevent output power bursts, that can result in receiver burst-errors or even damage to the receiver [3]. To avoid the cost of transient control in deeply saturated amplifiers, such as the erbium-doped fiber amplifier, a highly linear amplifier would, therefore, be an attractive candidate for future PON systems. Linear amplifiers based on gain-clamped semiconductor optical amplifiers (SOAs) [4] and combinations of SOAs and discrete Raman amplifiers [5] have recently been proposed for PON and metro applications. Although very good burst-mode performance has been demonstrated using these amplifiers, the

Manuscript received February 21, 2007; revised May 14, 2007.

R. Kjær, I. T. Monroy, L. K. Oxenløwe, and P. Jeppesen are with the $\mathrm{COM} \cdot \mathrm{DTU}$, Technical University of Denmark, DK-2800 Kgs. Lyngby, Denmark (e-mail: rkj@com.dtu.dk).

B. Palsdottir is with the OFS Fitel Denmark Aps, DK-2605 Brøndby, Denmark.

Color versions of one or more of the figures in this letter are available online at http://ieeexplore.ieee.org.

Digital Object Identifier 10.1109/LPT.2007.903768

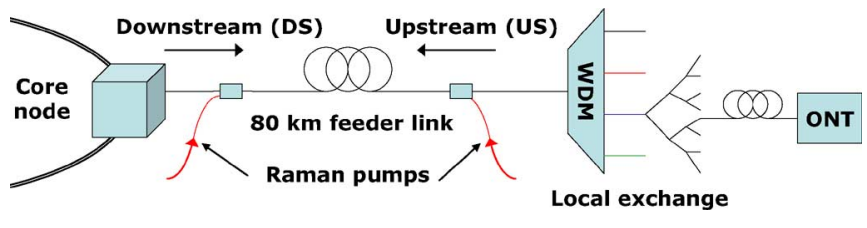

Fig. 1. Raman amplified WDM long-reach PON.

results have been limited to output powers up to $7.5 \mathrm{dBm}$, unrepeatered reach up to $60 \mathrm{~km}$, and unidirectional amplification.

Recently, a bidirectional link based on distributed Raman amplification (DRA) with $120 \mathrm{~km}$ of unrepeatered reach and symmetric channel bit rate of $10 \mathrm{~Gb} / \mathrm{s}$ was demonstrated for PON applications [6]. DRA was proposed for metro applications by Aoki et al. in [7] due to its highly linear gain properties and flexible gain bandwidth. Furthermore, DRA has excellent noise properties and offers bidirectional amplification on a single fiber. Fig. 1 shows an example of the proposed wavelength-division-multiplexed (WDM) long-reach PON, where bidirectional Raman pumping is used. In this scheme, amplification only takes place in the feeder link. In this letter, the system implications of burst-mode traffic in a bidirectionally pumped PON link is addressed and characterized for the first time. The dynamic performance of the link is quantified in terms of receiver sensitivity penalties, which are measured on a single surviving data channel. Two strong neighboring channels are periodically on-off modulated to "disturb" the surviving channel in both a co- and counterpropagating configuration and the number of disturbing channels is emulated by varying the input power of the disturbing channels. Finally, the observed performance difference in the case of co- and counterpropagating disturbance is explained using the measured output power transients.

\section{EXPERIMENTAL SETUP}

The experimental setup used to measure the bit-error rate (BER) and output power transients from the symmetric PON link is shown in Fig. 2. The link consists of $80 \mathrm{~km}$ of nonzero dispersion-shifted fiber (NZDSF), TrueWave RS, featuring low dispersion of $4.6 \mathrm{ps} / \mathrm{nm} / \mathrm{km}$ at $1550 \mathrm{~nm}$ and high Raman gain efficiency of $0.71 \mathrm{~W}^{-1} \cdot \mathrm{km}^{-1}$. The Raman pump light from two pump lasers is coupled into the link at each end using two WDMs. The WDMs and the fiber add up to a total link loss of $17.9 \mathrm{~dB}$. The pump wavelengths are chosen so they provide gain in the entire $C$-band and the total pump power is 233 and $221 \mathrm{~mW}$ in the co- and counterpropagating direction, respectively. The pumps provide a small-signal on-off gain of $22.0 \mathrm{~dB}$, corresponding to a small net gain of $4.1 \mathrm{~dB}$. The highly linear properties of the DRA-based link are confirmed by a high static $3-\mathrm{dB}$ input saturation power of $11 \mathrm{dBm}$. 


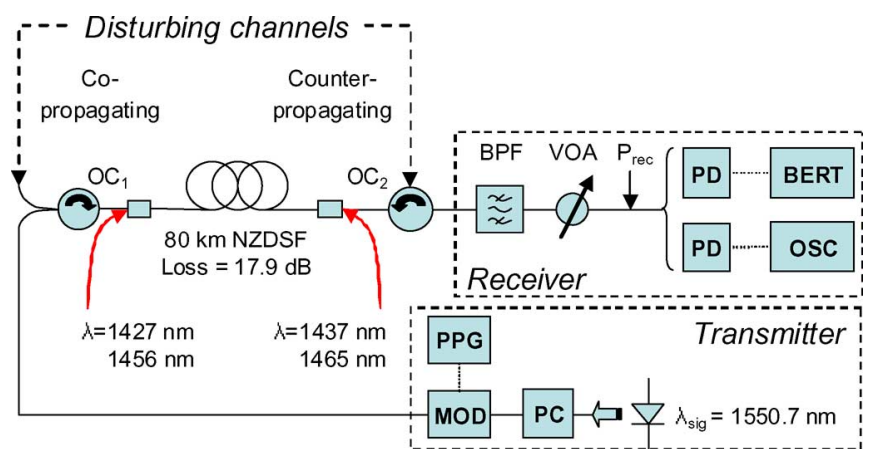

Fig. 2. Experimental setup used to measure BER and power transients.

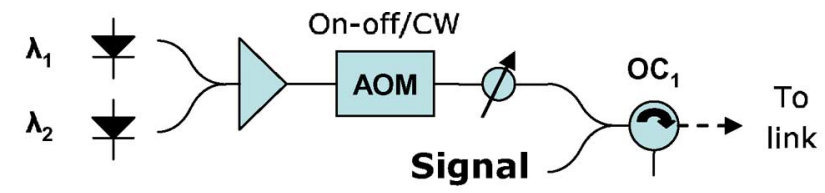

(a)

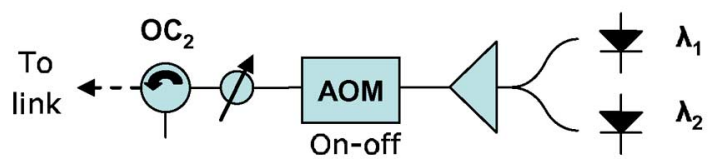

(b)

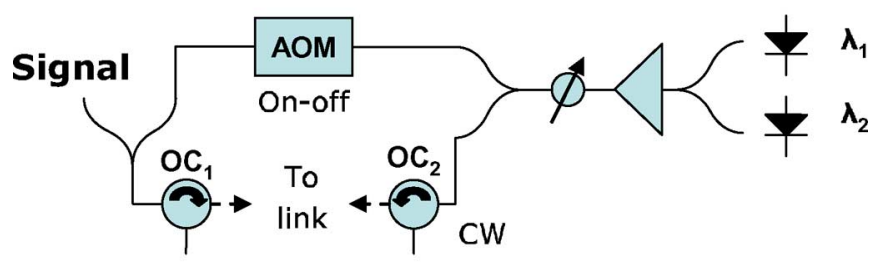

(c)

Fig. 3. Setups used to test three different cases of channel disturbance. (a) Copropagating; (b) counterpropagating; (c) copropagating with gain clamping.

A distributed feedback laser at $1550.7 \mathrm{~nm}$ is used as the data channel source. The polarization is optimized using a polarization controller (PC) before entering a Mach-Zehnder modulator (MOD), which is controlled by a pulse-pattern generator (PPG). A $10-\mathrm{Gb} / \mathrm{s}$ nonreturn-to-zero signal is encoded with a $2^{7}-1$ pseudorandom bit sequence. From here, the signal is combined with the disturbing channels using a $3-\mathrm{dB}$ coupler and fed into the link via an optical circulator $\mathrm{OC}_{1}$. The signal input power is fixed at $-2 \mathrm{dBm}$. It should be noted that upstream channels typically will have lower input powers, due to high splitting loss in the access network. A lower input power will generally reduce the amount of cross-gain modulation (XGM) and interchannel crosstalk. The receiver consists of a bandpass filter (BPF) followed by a variable optical attenuator (VOA) before the detector and BER test set (BERT). To characterize the transients, a low bandwidth (100 MHz) photodiode (PD) was used along with an oscilloscope.

The signal is disturbed using two neighboring channels positioned at $\lambda_{1}=1549.1 \mathrm{~nm}$ and $\lambda_{2}=1552.3 \mathrm{~nm}$. The disturbing channels modulate the saturable gain through XGM. The three test configurations are shown in Fig. 3. The channels are on-off modulated at $500 \mathrm{~Hz}$ with a $50 \%$ duty cycle using an acoustooptic modulator (AOM) with nanosecond response. The two channels have similar power levels and the sum of the channel

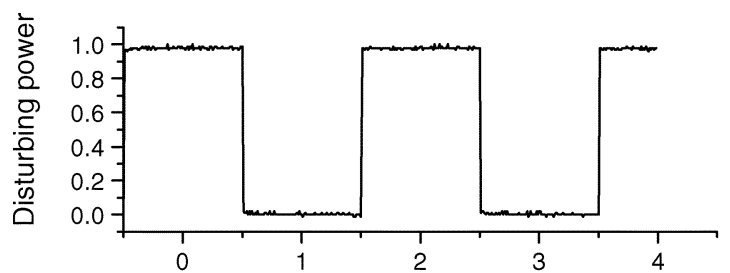

(a) Disturbing channels input

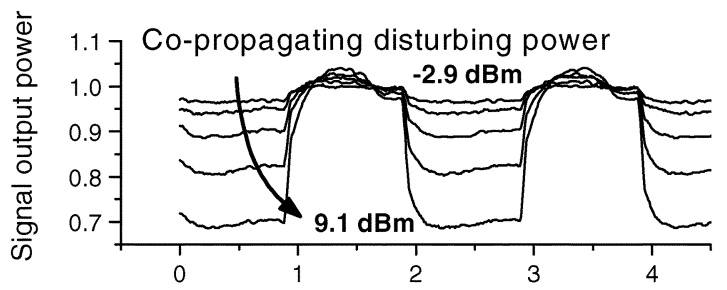

(b) Signal output with co-propagating disturbance

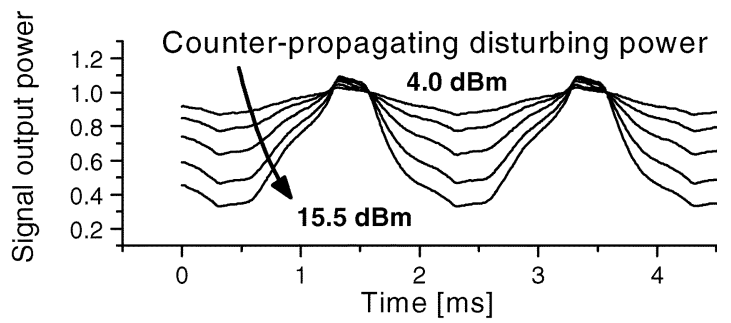

(c) Signal output with counter-propagating disturbance

Fig. 4. (a) Normalized disturbing power and (b) normalized signal output power in the case of co- and (c) counterpropagating disturbance.

powers into the span, the so-called disturbing power, is regulated using a VOA. In the cases where the disturbing channels are modulated, the disturbing power refers to the sum of the channel peak powers. In the first configuration in Fig. 3(a), the channels copropagate with the signal, while they counterpropagate with the signal in Fig. 3(b). In Fig. 3(c), some of the channel power is fed directly into the back of the link, resulting in counterpropagating continuous-wave $(\mathrm{CW})$, as well as a copropagating modulated, disturbance. In this configuration, the counterpropagating $\mathrm{CW}$ power is $4.5 \mathrm{~dB}$ higher than the copropagating peak power and thus has a "clamping" effect on the gain at high input powers.

\section{RESULTS AND DISCUSSION}

Fig. 4(a) shows the normalized link input power of the disturbing channels as a function of time. Fig. 4(b) and (c) shows the output power of the signal, in the case where the disturbing channels co- and counterpropagate with the signal, respectively. The levels are normalized so that unity output power corresponds to the unsaturated gain. Note that the maximum disturbance power used in Fig. 4(b) is $9.1 \mathrm{dBm}$ while the corresponding power in Fig. 4(c) is $15.5 \mathrm{dBm}$. There is a clear difference between the type of transients developed in the two cases. In the copropagating case, the power changes from saturated to unsaturated output power in the order of $\sim 400 \mu \mathrm{s}$. This time corresponds to the transit time of the $80-\mathrm{km}$ link. In the counterpropagating case, the transients develop over twice the transit time, i.e., close to $1 \mathrm{~ms}$. In both cases, small overshoots and undershoots can be observed. These are attributed to a small level of saturation imposed by the signal itself and the extra high pump depletion caused by the front end of the disturbing pulse. 


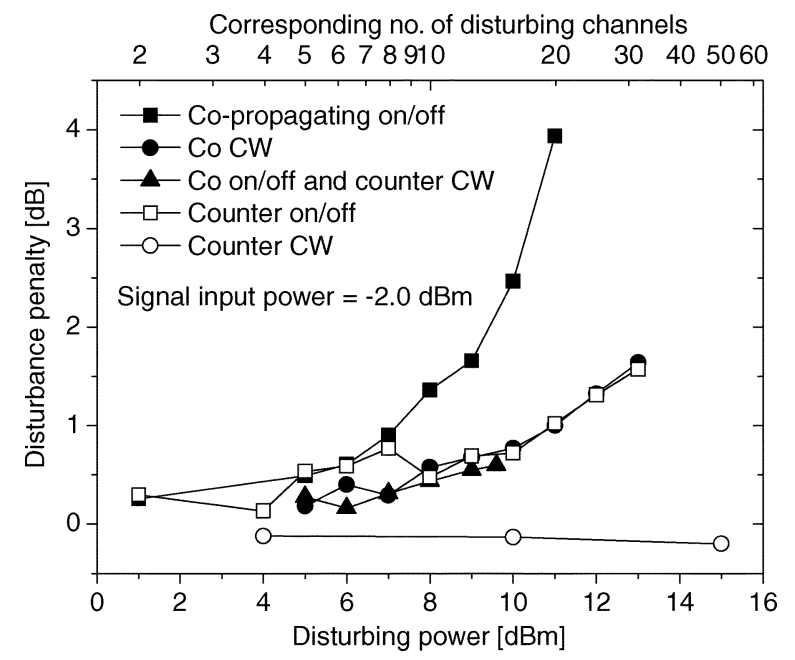

Fig. 5. Disturbance penalty, measured at a BER of $10^{-9}$, versus the total peak/CW power from two disturbing channels.

To measure the penalty imposed by the disturbing channels, the receiver sensitivity, at a BER of $10^{-9}$, is measured for different levels of disturbance power. Sensitivities of -20.4 and $-19.4 \mathrm{dBm}$ are found in the cases of back-to-back and after transmission with no disturbance, respectively. The disturbance penalty is defined as the sensitivity degradation due to the presence of the disturbing channels. Fig. 5 shows measurements of the disturbance penalty for the three configurations in Fig. 3. To estimate the penalty arising from different numbers of disturbing channels, the disturbance power is converted into a corresponding number of disturbing channels with $-2 \mathrm{dBm}$ of input power. This number is indicated by the upper axis in Fig. 5. When on-off disturbance is used (squares), penalties below $1 \mathrm{~dB}$ are found for disturbance powers up to 7 and $11 \mathrm{dBm}$, in the cases of co- and counterpropagating disturbance, respectively. These powers correspond to simultaneous on-off modulation of eight out of nine copropagating channels, or 20 out of 21 counterpropagating channels, respectively. The largest penalties are found when a copropagating on-off disturbance is used (solid squares). Here, the penalties reach a maximum value of $3.9 \mathrm{~dB}$ at a disturbance input power of $11.0 \mathrm{dBm}$. For higher disturbance powers, error-free detection was not possible due to severe eye closure in this configuration. In general, the penalty arising from on-off disturbance (squares) is larger than that arising from CW disturbance (circles). This can be attributed to the additional eye-closure contribution from the transient over- and undershoots observed in Fig. 4.

An important conclusion from Fig. 5 is that copropagating disturbance induces larger penalties than counterpropagating disturbance. In the $\mathrm{CW}$ case, no observable penalty is found for counterpropagating disturbance (hollow circles), while a 1.5-dB penalty is found for copropagating disturbance (hollow squares). The $\mathrm{CW}$ crosstalk is attributed to XGM mediated relative intensity noise (RIN) transfer from the disturbing channels. The reason for the higher tolerance toward counterpropagating disturbance is believed to be the following: The noise from the counterpropagating channels is averaged over the length of the fiber, thereby resulting in a low-pass filter transfer function, similar to the effect described by Fludger et al. in [8]. For small-signal fluctuations in long fibers, the $-3-\mathrm{dB}$ frequency will be of a few kilohertz [8]. Therefore, the higher frequency components of the RIN and the transient envelopes will not be transferred to the signal in the counterpropagating case. As a result, the penalty is lower in this case.

Fig. 5 (triangles) shows the penalties obtained when using the disturbance configuration displayed in Fig. 3(c). Here, the counterpropagating $\mathrm{CW}$ channels provide a moderate amount of gain clamping, which improves the penalty from the copropagating on-off disturbance (solid squares) by $\sim 1 \mathrm{~dB}$ at $9 \mathrm{dBm}$ of disturbance power. The result indicates that transient-induced penalties will be reduced in larger systems where the total bandwidth is distributed among many users and individual channel traffic will be more random.

\section{CONCLUSION}

Novel measurements of output power transients and the induced receiver sensitivity penalties, in a DRA-based PON link, have been presented. The burst-mode tolerance of the amplified link was tested on a single surviving channel with two co- or counterpropagating disturbing channels of varying input power. The highly linear performance of the amplified link was confirmed by penalties of less than $1 \mathrm{~dB}$ for co- and counterpropagating disturbance powers up to 7 and $11 \mathrm{dBm}$, respectively. Additional measurements showed that clamping the gain with two CW beams helped to reduce the penalty by more than $1 \mathrm{~dB}$. The improved penalty indicates that the burst-mode tolerance of the link should improve when the combined traffic is subject to statistical averaging in large networks (i.e., with many end-users). To conclude, we believe that the proposed DRAbased link should be regarded as a serious contender for future high-capacity links in PON networks with burst-mode traffic.

\section{REFERENCES}

[1] G. Talli and P. D. Townsend, "Feasibility demonstration of $100 \mathrm{~km}$ reach DWDM superPON with upstream bit rates of $2.5 \mathrm{~Gb} / \mathrm{s}$ and 10 $\mathrm{Gb} / \mathrm{s}$," in Proc. Optical Fiber Communication Conf. (OFC), Anaheim, CA, Mar. 2005, Paper OFI1.

[2] R. Davey, P. Healey, I. Hope, P. Watkinson, D. B. Payne, O. Marmur, J. Ruhmann, and Y. Zuiderveld, "DWDM reach extension of a GPON to 135 km," J. Lightw. Technol., vol. 24, no. 1, pp. 29-31, Jan. 2006.

[3] P. Lundquist, M. Levesque, J. Morrier, and D. Zaccarin, "Optical transients in cascaded EDFASs: Effects on transmission system performance," in Proc. Optical Amplifiers and Their Applications (OAA), Otaru, Japan, 2003, Paper MD03.

[4] N. Suzuki and J. Nakagawa, "First demonstration of full burst optical amplified GE-PON uplink with extended system budget of up to 128 ONU splits and $58 \mathrm{~km}$ reach," in Eur. Conf. Optical Communication (ECOC), Glasgow, U.K., Sep. 2005, Paper Tu1.3.3.

[5] H. H. Lee, J. M. Oh, D. Lee, G. W. Lee, and S. T. Hwang, "Performance of $16 \times 10 \mathrm{~Gb} / \mathrm{s} \mathrm{WDM}$ transmissions over $4 \times 40 \mathrm{~km}$ of SMF using linear optical amplifiers combined with Raman-pumped dispersion compensation fibers under dynamic add-drop situations," IEEE Photon. Technol. Lett., vol. 16, no. 6, pp. 1576-1578, Jun. 2004.

[6] R. Kjær, I. T. Monroy, B. Palsdottir, L. K. Oxenløwe, and P. Jeppesen, "Bi-directional $120 \mathrm{~km}$ long-reach PON link based on distributed Raman amplification," in Proc. IEEE Lasers and Electro-Optics Society Annual Meeting (LEOS'06), Montreal, Canada, Oct. 2006, Paper WEE3.

[7] Y. Aoki and S. Kinoshita, "Transient response characteristics of Raman amplified optical add/drop multiplexing network," Proc. SPIE, vol. 4872, pp. 129-136, 2002.

[8] C. R. S. Fludger, V. Handerek, and R. J. Mears, "Pump to signal RIN transfer in Raman fiber amplifiers," J. Lightw. Technol., vol. 19, no. 2, pp. 1140-1148, Feb. 2001. 